BULLETIN OF THE

AMERICAN MATHEMATICAL SOCIETY

Volume 79, Number 4, July 1973

\title{
SOLOMON LEFSCHETZ AN APPRECIATION IN MEMORIAM
}

\author{
BY LAWRENCE MARKUS
}

Solomon Lefschetz began and ended his scientific career as a theoretical engineer. In between, he accomplished the work of several lifetimes of creative foundational research in algebraic geometry and topology, complemented by important contributions to such diverse fields of mathematics as differential equations, control theory, and nonlinear mechanics. In addition to his fundamental mathematical discoveries and authoritative expositions, the influence of Professor Lefschetz will long be spread by the mathematical organizations he established, and the students of all levels he inspired by his courageous enthusiasm, humane leadership, and critical scholarship.

Born in Moscow on 3 September 1884, graduate engineer at Ecole Centrale of Paris in 1905, mathematical doctorate from Clark University in 1911, Professor of Mathematics at Princeton from 1925-1953, President of the American Mathematical Society 1935, visiting Professor at the Center for Dynamical Systems at Brown University after 1964, died in Princeton on 5 October 1972; the personal data of the life of Solomon Lefschetz can be read from biographical sources such as The New York Times 7 October 1972, or the World Who's Who in Science 1968. In this brief note we can only mention some of his most famous mathematical achievements and comment, from personal knowledge, on some of the remarkable scientific activities and profound influences flowing from his later life.

The best source for understanding the significance of the mathematical work of Lefschetz lies in his own writings. In 1971 he edited a volume, Selected papers [136], of his major mathematical papers and monographs, including a complete bibliography through 1969 to which the final entries have been added below. Of the eighteen articles in the Selected papers the first several deal with global analysis and topology of algebraic varieties. These include the famous paper,

On certain numerical invariants of algebraic varieties, with applications to abelian varieties [24] (Awarded Prix Bordin of the Paris Academie des Sciences 1919, and Bôcher Memorial Prize of American Mathematical Society 1924),

the extensive monograph in the Borel Series, 
L'Analysis situs et la géométrie algebrique [29],

as well as the reflective review article of 1968 ,

A page of mathematical autobiography [130].

The problems that Lefschetz solved concerned the determination of the number of independent meromorphic differential $p$-forms of various kinds on a nonsingular algebraic variety $V^{n}$, and the relation of these numbers to the Betti numbers $R_{p}$ of the integral homology groups of $V^{n}$. Lefschetz writes a half-century later $[130]$ :

'It was the general implicit or explicit understanding among algebraic geometers of my day that an algebraic $n$-variety $V^{n}$ ( $n$ dimensional variety) is the partial or complete irreducible intersection of several complex polynomials or "hypersurfaces" of a projective space $S^{n+k}$, in which $V^{n}$ had no singularities (it was homogeneous). Thus $V^{n}$ was a compact real 2n-manifold $M^{2 n}$ (complex dimension $n$ ) ...

Incidentally, the recent brilliant reduction of singularities by Hironaka has shown that the varieties as just described are really entirely general.'

Thus a nonsingular algebraic variety $V^{n}$ is a compact complex submanifold, defined by polynomial equations in some complex projective space. Hence $V^{n}$ is itself a complex manifold (in fact, a Kähler manifold) with holomorphic local coordinate charts, say $\left(z^{1}, z^{2}, \ldots, z^{n}\right)$, in terms of which one can define holomorphic and meromorphic complex functions and differential forms.

For $n=1, V^{1}$ is an algebraic curve or Riemann surface. In this case the theory of abelian integrals $\int R(z, y) d z$ belongs to classical function theory. Here the meromorphic differential 1-form $\omega^{1}=R(z, y) d z$, where $R(z, y)$ is a rational function of the complex variable $z$ and of the algebraic function $y(z)$ defining $V^{1}$. Riemann showed that the number of holomorphic differentials (linearly independent over the complex field) is just the genus of the curve $V^{1}$.

On $V^{n}$ the differential $p$-forms (or integrals) under discussion, all assumed to be everywhere meromorphic, can be written in local coordinates $\omega^{p}=\sum \alpha_{j_{1}, \ldots, j_{p}}(z) d z^{j_{1}} \ldots d z^{j_{p}}$. We say that $\omega^{p}$ is

(a) of the first kind when it is holomorphic everywhere on $V^{n}$,

(b) of the second kind if, in the neighborhood of any point, it differs from a locally holomorphic $p$-form by the exterior derivative of a $(p-1)$ form on $V^{n}$.

A $p$-form $\omega^{p}$ is closed if $d \omega^{p}=0$ on $V^{n}$, and $\omega^{p}$ is exact if it equals the exterior derivative of some $(p-1)$-form. Two $p$-forms are to be regarded as equivalent if their difference is exact. The main problem is to determine 
the complex vector space $\mathscr{V}^{q}$ of equivalence classes of closed $p$-forms, of first and second kinds, on an algebraic variety $V^{n}$.

For the case of an algebraic surface $\mathscr{V}^{2}$ Lefschetz proved ([130] or $[136$, pp. 25, 33]) (the first statement clarifying earlier results of Picard and Poincaré, and the second exhibiting his principal results for surfaces)

closed 1-forms of the first kind make up a $\mathscr{V}^{q}$ with $q=\frac{1}{2} R_{1}$,

and

closed 2-forms of the second kind make up a $\mathscr{V}^{\rho_{0}}$ and the basic formula holds,

$$
\rho_{0}=R_{2}-\rho,
$$

where $\rho$ is the Betti number of algebraic 2-cycles.

The procedure of computing $\rho$, the number of independent algebraic 2-cycles, is implemented by the result that an algebraic 2-cycle is precisely an algebraic curve (using virtual curves and algebraic dependence in the sense of Severi). Lefschetz extends his results to general varieties $V^{n}$ and, in particular, clarifies the role of algebraic $(2 n-2)$-cycles in the theorem ([130] or $[136$, p. 38]),

for hypersurfaces of $V^{n}$ algebraic dependence and homology in $V^{n}$ are equivalent relations.

For the topology of algebraic varieties the results strike one as even more sensational (see Hodge [1A]), although the mystery was afterwards somewhat exorcised through the theory of harmonic forms on Kähler manifolds.

For an algebraic variety $V^{n}$ the odd-dimensional Betti numbers are even, and furthermore $R_{p} \geqq R_{p-2}$ for $2 \leqq p \leqq n$.

Thus, not every orientable compact 4-manifold can be an algebraic surface or, as Lefschetz asserts in his Borel Tract,

'Les surfaces et les variétés algebriques accusent, au point de vue de l'Analysis Situs, des différences profondes avec les courbes, différences dues surtout à ce qu'elles ne sont pas les variétés bilatères les plus generales de leur dimension (quatre pour les surfaces, $2 \mathrm{~d}$ pour les varietés à $d$ dimensions).'

To understand the method of approach to these theorems, and their significance to further mathematics we are fortunate to be able to depend on two remarkable expositions, 
A page of mathematical autobiography, by S. Lefschetz [130], and

Professor Lefschetz's contributions to algebraic geometry: an appreciation, by W. V. D. Hodge [1A].

The second item appeared in connection with a Symposium in Algebraic Geometry and Topology at Princeton University in 1954 in honor of Professor Lefschetz on his seventieth birthday.

Today when the language of algebraic geometry, involving sheaves and schemes, seems remote from the interests of most mathematicial analysists, it is difficult to recall that a couple of generations ago this subject concerned the evaluation of line and surface integrals of multivalued functions in the complex domain.

'In its early phase (Abel, Riemann, Weierstrass), algebraic geometry was just a chapter in analytic function theory' $([130]$ or $[136, p .14])$.

Lefschetz approached this subject via the classical route, following Picard, but using some of the elements of projective geometry and combinatorial topology introduced by Riemann and Poincaré.

'One of the first applications of his work on the topology of algebraic varieties which Lefschetz made was to the theory of integrals of the second kind. Some of his work on this subject preceded the work on the topology of varieties, and it seems fairly clear that he was led to the topological work in order to make progress possible in the study of integrals' [1 $\mathrm{A}]$.

Even in his text Algebraic geometry [93], written in the modern algebraic style in 1953, Lefschetz writes

'At all events one cannot write on algebraic geometry today outside of the general framework of algebra. On the other hand many have come to algebraic geometry and have been attracted to it through analysis, and it would seem most desirable to preserve this attraction and this contact.'

An insight into the direct and bold methods employed by Lefschetz is available in the following sections of the autobiographical sketch [130]:

$\S 7$ "Certain properties of the surface $F$. Its characteristic", and

$\S 8$ "One-cycles of $F$ ".

In this description Lefschetz considers the algebraic surface $V^{2}=F$ represented in a cartesian space of 3-complex variables by

$$
F(x, y, z)=0
$$

which is in general position relative to the axes. For each fixed value of 
$y=$ const, let $H_{y}$ be the hyperplane-section in the surface $F$. In general $H_{y}$ is an algebraic curve (a Riemann surface of an algebraic function of one variable), excepting a finite set of planes $y=a_{k}$ which are tangent to $F$. Then Lefschetz concludes

I. Every $H_{y}, y$ not an $a_{k}$, is of fixed genus $p$.

II. Every $H_{y}$ is irreducible.

III. The plane $y=a_{k}$ has a unique point of contact $A_{k}$ with $F$, and $A_{k}$ is a double point of $H_{a_{k}}$ with distinct tangents. Hence the genus of $H_{a_{k}}$ is $p-1$.

With this geometric picture, Lefschetz proceeds to make an explicit cellular decomposition of $H_{y}$, and then of $F$, to compute the Euler characteristic of the surface $F$.

The next step was to compute the number of independent (in the sense of homology $\sim$ ) 1 -cycles in order to calculate the Betti number $R_{1}(F)$. Picard had proved that every 1-cycle $\gamma^{1}$ of $F$ is homologous to a 1-cycle contained in a section $H_{y}$. Also $H_{y}$ contained a certain number $r$ of 1cycles which are invariant as $y$ varies. That is, such a cycle $\gamma^{1}$ situated say in $H_{a}$ (for $a \neq a_{k}$ ) has the property that as $y$ describes any closed path from $a$ back to $a$ on the Riemann sphere $S_{y}$, the cycle $\gamma^{1}$ returns to a cycle $\sim \gamma^{1}$ in $H_{a}$.

On the other hand as $y$ describes a path $a a_{k}$ on $S_{y}$ a certain 1-cycle $\delta_{k}^{1}$ of $H_{a}$ tends to the point of contact $A_{k}$ and hence is $\sim 0$ on $H_{a_{k}}$. This is the vanishing cycle as $y \rightarrow a_{k}$. Using these vanishing cycles $\delta_{k}^{1}$, and their intersection multiplicities, or Kronecker indices $\left(\gamma^{1}, \delta_{k}^{1}\right)$, with other 1-cycles on $H_{a}$, Lefschetz proves $([130]$ or $[136$, p. 22])

THEOREM. N.a.s.c. for invariance of the cycle $\gamma^{1}$ is that every $\left(\gamma^{1}, \delta_{k}^{1}\right)=0$; and then the desired result for the first Betti number of the surface $F$,

THEOREM. The number of invariant cycles of $H_{y}$ is equal to the Betti number $R_{1}(F)$ and both are even: $r=R_{1}=2 q$.

To summarize the significance of these methods and results in algebraic geometry we turn to the Appreciation by Hodge [1A]:

'Moreover, Lefschetz's work is the direct inspiration of all researches which have taken place subsequently in the theory of complex manifolds. In fact, it is not too much to say that our greatest debt to Lefschetz lies in the fact that he showed us that a study of topology was essential for all algebraic geometers ...

To speculate on what might have been, had some historical event not taken place, is a singularly useless occupation, and any opinion on how 
algebraic geometry would have developed without Lefschetz's intervention can only be a personal one. I am, however, in a position to state one incontrovertible fact. The idea of generalizing the notion of an algebraic integral to give a theory of harmonic integrals on an algebraic variety arose out of a study of Chapter IV of Lefschetz's Borel Tract, and an attempt to carry the work of that chapter further, and but for the influence that Tract had on me I should never have thought of the idea...

No single person can claim to be the sole founder of the theory of complex manifolds, but when one considers how many of the properties derived by Lefschetz for algebraic varieties now hold their place in the theory of complex manifolds, and how he influenced decisively so many who have contributed to this theory, one must accord him an honored place among the founders of a great branch of mathematics; and this without even taking account of his influence, through his work in pure topology, on the topologists who have helped to build the theory. It seems clear to me that Lefschetz by his work on the topology and transcendental theory of algebraic varieties has been a major influence in turning the minds of geometers in new and fruitful directions, and in so doing he has achieved what it is given to few to do.'

And finally Lefschetz himself reviewed his contribution to algebraic geometry $[\mathbf{1 3 0}]$ :

'As I see it at last it was my lot to plant the harpoon of algebraic topology into the body of the whale of algebraic geometry.'

The main contributions of Lefschetz to algebraic topology were his fixed point theorem for manifolds, and the development of the algebraic machinery of singular chain complexes, relative homology, and duality theory necessary to obtain the corresponding fixed point formula for general locally connected spaces. Thirteen articles in his Selected papers chart this route. Through all this work, culminating in the Colloquium Publication of 1942 [79], Algebraic topology, the common thread is the interest in the intersection properties of cycles. In this sense the topological researches of Lefschetz flowed uninterruptedly from his studies of the intersection properties of the vanishing cycles on algebraic surfaces.

In his two famous papers of 1926 and 1927, Intersections and transformations of complexes and manifolds [33], and Manifolds with a boundary and their transformations [36], Lefschetz obtains his fixed point theorem as a special result within his theory of coincidences of cycles. In the first of the two papers, mainly devoted to the proof of the fixed point theorem for compact orientable manifolds without boundaries, Lefschetz writes

'The principle of the method is best explained by means of a very simple 
example. Let $f(x)$ and $\varphi(x)$ be continuous and uni-valued functions over the interval $[0,1]$, and let their values on the interval also be between 0 and 1 . It is required to find the number of solutions of $f(x)=\varphi(x)$, $0 \leqq x \leqq 1$.

Graphically the problem is solved by plotting the curvilinear arcs

$$
y=f(x), \quad y=\varphi(x), \quad 0 \leqq x \leqq 1,
$$

and taking their intersections. A slight modification of the functions may change the number of solutions, even make them become infinite in number. However, the difference between the numbers of positive and negative crossings of sufficiently close polygonal approximations to the arcs is a fixed number, their Kronecker index. Its determination is then a partial answer to the question, and indeed seemingly the only possible general answer.

The two complexes whose product is taken in this case are the unit segments on the $x$ and $y$ axes, their product being the square whose sides they are. Replace the unit segments by two identical manifolds of $n$ dimension, $M_{n}$ and $M_{n}^{\prime}$, the square by the $M_{2 n}$ image of their pairs of points (product of the two), the arcs by the manifolds on $M_{2 n}$ and the exact situation of Part II is obtained.'

Here Lefschetz counts the number of coincidences of the maps $f(x)$ and $\varphi(x)$ by the intersection multiplicity or Kronecker index of their graphs in the unit square. If $\varphi(x)=x$ is the identity map, then the fixed points of $f(x)$ are obtained as solutions of $f(x)=x$ on $0 \leqq x \leqq 1$. There are two failures of this example to illustrate the full approach to the general case where $f$ is a continuous map of a compact orientable manifold $M_{n}$ into itself:

(a) The unit segment $[0,1]$ is not a cycle as is $M_{n}$, since it has a nonempty boundary, and the intersection theory of cycles is not directly applicable.

(b) The unit segment $[0,1]$ has dimension 1 , and higher dimensional spaces require deeper insight.

The first difficulty can be bypassed for the moment by replacing $[0,1]$ by the circle $S^{1}$, the square by the torus $T^{2}=S^{1} \times S^{1}$, and demanding that $f$ and $\varphi$ satisfy suitable periodicity conditions. In this case the "average slopes" of the graphs of $f$ and $\varphi$ in the plane (using $\boldsymbol{R}^{2}$ as the covering space of $T^{2}$ ), are significant and these can be expressed in terms of the induced homomorphisms of the homology group $H_{1}\left(S^{1}\right)=Z$. The second difficulty is essentially the heart of the Lefschetz analysis, and homology at all dimensions must be considered. In the 1926 paper Lefschetz develops the fixed point theory for orientable manifolds without boundary, and in the paper of 1927 he extends his theory to manifolds with boundary using relative homology groups. He phrases his fixed-point 
criterion in terms of an integral number $L(f)$, which reduces to the Eulercharacteristic of the space $X$ when $f$ is homotopic to the identity map, that is, when $f$ is a deformation of $X$.

For an exposition of the precise results we turn to an impressive article by N. E. Steenrod [1A]:

The work and influence of Professor S. Lefschetz in algebraic topology.

'The basic step toward a full-fledged result (on fixed point theory) was Lefschetz's discovery in 1923 of a formula. Its description runs as follows. Let $f$ be a continuous map of a topological space $X$ into itself. For each dimension $n, f$ induces an endomorphism $f_{n}$ of the homology group $H_{n}(X)$ based on the rational numbers $R$ as coefficient group. Now $H_{n}(X)$ is a vector space over $R$. If its rank is finite, there is assigned a numerical invariant of $f_{n}$ called its trace and denoted by $\operatorname{Tr}\left(f_{n}\right)$. The trace is computed by choosing a base for $H_{n}$ and taking the trace of the corresponding matrix representation of $f_{n}$. Then the Lefschetz number of $f$, denoted by $L(f)$, is given by

$$
L(f)=\sum_{n=0}^{\infty}(-1)^{n} \operatorname{Tr}\left(f_{n}\right) .
$$

It is clear that restrictions must be imposed if $L(f)$ is to be well defined. It suffices, for example, to require that $X$ be the space of a finite complex. Then it can be shown that $\operatorname{Tr}\left(f_{n}\right)$ is an integer, and it is zero in dimensions exceeding that of the complex; hence $L(f)$ is defined and is an integer.

The conclusion of the fixed point theorem reads: If $L(f) \neq 0$, then $f$ has at least one fixed point (i.e. there is a point $x \in X$ such that $f(x)=x$ ).

The conclusion is valid whenever $X$ is the space of a finite complex. This result was proved by Lefschetz in 1928 .

His initial theorem in 1923 asserted the conclusion only when $X$ is a compact orientable manifold (without boundary)...

Although the fixed-point theorem for manifolds is an extremely beautiful result, Lefschetz must have been dissatisfied by the fact that it did not include the fixed-point theorem of Brouwer for an $n$-cell. A cell is not a manifold. However, it is a manifold with boundary which is itself a manifold, i.e. it is a relative manifold. If the techniques used in proving the fixed-point formula for manifolds could be extended to relative manifolds, then the Brouwer theorem might be included. The techniques in question were products, intersections, and duality ...

Successive papers marked successive steps in the process from closed manifolds to relative manifolds, to general complexes, to the final form for locally connected spaces. In consequence he was a central participant 
in one of the major trends of the period 1925-1935, namely, the extension of the methods of combinatorial analysis situs to general topological spaces.

It is indicative of the influence of Lefschetz that the present-day usage of the terms "topology" and "algebraic topology" is due to him. Before the appearance in 1930 of his first Colloquium Publication entitled Topology, the subject was known as analysis situs. When his second Colloquium Publication entitled Algebraic topology appeared in 1942, the adjective "combinatorial" fell into disuse.'

Following his extensive research activities in algebraic geometry and algebraic topology, Lefschetz entered a third scientific field with the publication of a monograph Lectures on differential equations [84] in 1946. He had been led to renew his early engineering interests in mechanics by his associations with various scientists working on electronic and mechanical guidance systems during World War II. He was further stimulated by the serious Soviet activities in this direction, and during the subsequent decade he translated a number of Russian monographs and important articles into English. The influence of Lefschetz (with R. Courant and a very few others), in encouraging young mathematicians interested in the theory and applications of differential equations, and in making applied mathematics respectable and even important within the American Mathematical Society, can hardly be over-estimated. In a volume [3A] of the Journal of Mathematical Analysis and Applications 1965, dedicated to Lefschetz, the editors R. Bellman and J. P. LaSalle state

'The achievements of Solomon Lefschetz in the field of topology and in the development of American mathematics in general are well known. What is perhaps not so well known is that, in 1944, at the age of sixty, he began a new career in the field of differential equations and control theory. With his remarkable insight and intuition he saw the mathematical possibilities in these areas. With his indefatigable energy and enthusiasm he put together, first at Princeton University then at RIAS, and now at Brown University, outstanding research groups in these domains. He also pursued this research vigorously at the University of Mexico as a professor of mathematics after his retirement from Princeton.

On behalf of his many pupils and colleagues, and many more friends, we would like to express our appreciation of his mathematical genius, intellectual courage, and broad humanity in dedicating this volume to him.'

In Contributions to the theory of nonlinear oscillations ([88], [91], [106]), a set of volumes which he edited in the series of Princeton Annals Studies, Lefschetz sets out his two main interests in the field of dynamical systems: 
(a) the general theory of dissipative systems including the concept of structural stability,

(b) the algorithmic approach to the resolution of singularities of critical points and bifurcating periodic orbits.

The first of these two programs (a) is emphasized in the first volume of the Contributions in 1950:

'Nonlinear conservative oscillators have been investigated mainly in connection with celestial mechanics, and the information available for them is therefore rather extensive. It is known, for example, that the trajectories are extremals of a variational problem, so that one may bring to bear upon the problem Morse's technique for the discovery of closed geodesics on manifolds. Nothing of this sort is at hand for the dissipative type, making progress slow.'

Lefschetz had been attracted to the theory of dissipative (as distinct from conservative) dynamical systems since these are of central importance in engineering problems where friction and resistance are significant. Also such dynamical systems, which can be interpreted mathematically as vector fields on the phase-space manifold, are amenable to the techniques of homotopy (a damped oscillator is qualitatively unchanged if the damping coefficient is slightly varied). In the concept of structurally stable systems, indicated in a note by Pontryagin and Andronov, Lefschetz found the direction he was seeking.

On a differentiable manifold $M$, say compact and without boundary, consider the collection $\Sigma$ of all $C^{1}$-vector fields, and endow $\Sigma$ with the $C^{1}$-topology. Define two systems $S_{1}$ and $S_{2}$ of $\Sigma$ to be qualitatively equivalent in case there exists a homeomorphism of $M$ onto itself throwing all the (unparametrized) solution curves of $S_{1}$ onto those of $S_{2}$. Then a dynamical system $S$ is called structurally stable in case there is a neighborhood $N$ of $S$ in $\Sigma$ such that each $S_{1} \in N$ is qualitatively equivalent to $S$.

It is clear that structurally stable differential systems should be of fundamental importance in engineering, biological, and social dynamics where the qualitative features must be predicted without absolutely accurate knowledge of the parameters of the physical phenomena.

Lefschetz guided and encouraged students and young mathematicians on his projects to work on these qualitative problems of global analysis. In particular, his thesis student H. F. DeBaggis clarified the results of Pontryagin for the sphere $S^{2}=M$, and M. Peixoto proved that the structurally stable systems on a compact surface $M^{2}$ constitute an open dense subset of $\Sigma$. This reporter continued these studies on arbitrary manifolds $M^{n}$ and proved that a structurally stable system must have isolated and elementary critical points and periodic orbits. Then S. Smale and his 
associates obtained the brilliant result that every Morse-Smale system (defined by certain elementary structural conditions) is structurally stable on $M^{n}$. Thus the path illuminated by Lefschetz became one of the main routes of research for dynamical system theory during the two decades $1950-1970$, and his expectations were fully justified.

The second problem (b) suggested by Lefschetz was attacked by him in a series of papers written at the University of Mexico. Also the single article [97] on differential equations that appears in his Selected papers is in this area. Here the emphasis is on a detailed, almost algorithmic, analysis of all possible structures for degenerate equilibrium points and periodic orbits of a dynamical system. The mathematical tools are those of the Weierstrass Preparation Theorem and Puiseux fractional power series. Clearly these methods stem from Lefschetz's early interests in the resolution of singularities in algebraic geometry.

Lefschetz exercised an enormous, and beneficial, influence among the post-war generation of mathematicians in the fields of dynamical systems, nonlinear mechanics, and control theory. A glance through the list of authors in the Contributions (edited by Lefschetz), ([88], etc.) or the Proceedings of the symposium at Colorado Springs (organized by Lefschetz) [121], or the Symposium at Puerto Rico (dedicated to Lefschetz) [2A] gives a rather complete view of mathematicians in these fields in the United States (of America and of Mexico) and Western Europe.

Even after the age of eighty Lefschetz continued to explore new directions in mathematics and his creative and organizational talents were turned towards nonlinear control theory. In the Symposium in Puerto Rico he states

'The most interesting and most recent application of Liapunov's theory is to the stability of nonlinear controls. There is, of course, an extensive theory of linear controls, stability included, whose origin goes back to Maxwell and Vishnegradskii about a century ago. With the advance of modern technology however nonlinear schemes and in particular nonlinear controls have appeared, calling inevitably for Liapunov's theory.'

Lefschetz attacked technical engineering problems, not rarefied mathematical generalities. For instance he made significant contributions to the Lurie stability problem, so named after the Soviet Academician. To understand the nature of this nonlinear control stability problem, let us first look at the linear case.

Consider a real vector differential system

$$
d x / d t=A x-b \xi
$$

where $A$ is an $n \times n$ constant matrix and $b$ is a constant column $n$-vector. 
The state of the system is the $n$-vector $x$ at each time $t$, and the control $\xi$ is a scalar. An important problem of linear control theory consists in choosing $\xi=c x, \xi$ as a linear function of $x$ using the constant row vector $c$, so that the resulting feedback system

$$
\dot{x}=(A-b c) x
$$

is asymptotically stable towards the null solution, that is, each solution $x(t) \rightarrow 0$ as $t \rightarrow+\infty$.

Let us now keep the above linear dynamics $\dot{x}=A x-b \xi$ but use nonlinear feedback control laws $\xi=\varphi(c x)$ for a real $C^{1}$-function $\varphi(\sigma)$ satisfying

$$
\sigma \varphi(\sigma)>0 \text { for } \sigma \neq 0, \text { and } \int_{0}^{ \pm \infty} \varphi(\sigma) d \sigma=+\infty .
$$

This is the direct method of control, generalizing the linear control law $\varphi(\sigma)=\sigma$, as described in the text [122] by Lefschetz.

A more interesting engineering problem was introduced by Lurie, using the linear dynamics $\dot{x}=A x-b \xi$ and the "indirect or differential control" $\dot{\xi}=\varphi(c x-\rho \xi)$. This control system is called absolutely stable in case every solution $x(t) \rightarrow 0, \xi(t) \rightarrow 0$ as $t \rightarrow+\infty$, for every admissible control law $\varphi(\sigma)$, satisfying the above conditions. Thus, given $A$ and $b$, the problem is to find all vectors $c$ and scalars $\rho>0$ such that the resulting system is absolutely stable. Lefschetz gave an explicit and useful stability criterion. In the special, but important, case where

$$
A=\operatorname{diag}\left\{\lambda_{1}, \lambda_{2}, \ldots, \lambda_{n}\right\} \quad \text { with } \lambda_{h}<0,
$$

Lefschetz's criterion [122] reduced to the simple form

$$
\rho>\sum_{h=1}^{n}\left|\frac{b_{h} c_{h}}{\lambda_{h}}\right|
$$

(moreover, various improvements are also offered there, for instance, the sum can omit all terms for which $b_{h} c_{h} \geqq 0$ ).

Professor Lefschetz was an extraordinarily stimulating and fascinating personality. At the age of eighty he was still actively lecturing at three universities, Princeton, Mexico, and Brown, and was continuing his researches in algebraic geometry, topology, and dynamical systems. He had received highest honors from many governments, the National Medal of Science (United States), the Order of the Aztec Eagle (Mexico) and he held membership in the major scholarly societies, National Academy of Sciences (United States), l'Academie des Sciences de Paris, Royal Society of London, among others.

He was, of course, a brilliant linguist speaking and writing frequently 
in Russian, French, English, and Spanish (he once remarked that he considered all European tongues to be dialects of a single language). But he would sometimes surprise the company by conversing in Persian, or some other distant language. It was not unusual for him to present a Symposium address in English and then repeat the lecture or its abstract immediately in Russian or Spanish to the delight of the audience.

His instantaneous translations of other lectures could be amusing and startling. At a Symposium at Colorado Springs Lefschetz was asked to translate a steady half-hour torrent of Russian. He did so in one sentence:

He says, "you try to get the final formula from the first one."

This direct approach was always his philosophy, and in intellectual problems and in human issues Solomon Lefschetz was eminently successful at getting the final formula.

\section{BIBLIOGRAPHY FOR SOLOMON LEFSCHETZ}

[1] Two theorems on conics, Ann. of Math. (2) 14: 47-50.

[2] On the $V_{3}^{3}$ with five nodes of the second species in $S_{4}$, Bull. Amer. Math. Soc. 18: $384-386$.

[3] Double curves of surfaces projected from space of four dimensions, Bull. Amer. Math. Soc. 19: 70-74.

1913

[4] On the existence of loci with given singularities, Trans. Amer. Math. Soc. 14: 23-41; Doctoral Dissertation, Clark University, Worcester, Mass., 1911.

[5] On some topological properties of plane curves and a theorem of Möbius, Amer. J. Math. 35: 189-200.

1914

[6] Geometry on ruled surfaces, Amer. J. Math. 36: 392-394.

[7] On cubic surfaces and their nodes, Kansas Univ. Science Bull. 9: 69-78. 1915

[8] The equation of Picard-Fuchs for an algebraic surface with arbitrary singularities, Bull. Amer. Math. Soc. 21: 227-232.

[9] Note on the $n$-dimensional cycles of an algebraic n-dimensional variety, Rend. Circ. Mat. Palermo 40: 38-43.

1916

[10] The arithmetic genus of an algebraic manifold immersed in another, Ann. of Math. (2) 17: 197-212.

[11] Direct proof of De Moivre's formula, Amer. Math. Monthly 23: 366-368.

[12] On the residues of double integrals belonging to an algebraic surface, Quart. J. Math. 47: 333-343.

1917

[13] Note on a problem in the theory of algebraic manifolds, Kansas Univ. Science Bull. 10: $3-9$.

[14] Sur certains cycles à deux dimensions des surfaces algébriques, Accad. Lincei. Rend. (5) 26: 228-234.

[15] Sur les intégrales multiples des variétés algébriques, C. R. Acad. Sci. Paris 164: $850-853$.

[16] Sur les intégrales doubles des variétés algébriques, Ann. di Mat. (3) 26: 227-260. 1919

[17] Sur l'analyse situs des variétés algébriques, C. R. Acad. Sci. Paris 168: 672-674.

[18] Sur les variétés abéliennes, C. R. Acad. Sci. Paris 168: 758-761.

[19] On the real folds of Abelian varieties, Proc. Nat. Acad. Sci. U.S.A. 5: 103-106.

[20] Real hypersurfaces contained in Abelian varieties, Proc. Nat. Acad. Sci. U.S.A. 5: $296-298$. 
1920

[21] Algebraic surfaces, their cycles and integrals, Ann. of Math. (2) 21 : 225-228; correction, ibid. 23: 333 .

[22] Quelques remarques sur la multiplication complexe, Comptes Rendus du Congrès International des Mathématiciens (Strasbourg, Sept. 1920), É. Privat, Toulouse, 1921, pp. 300-307.

[23] Sur le théorème d'existence des fonctions abéliennes, Accad. Lincei. Rend. (5) 30: $48-50$.

*[24] On certain numerical invariants of algebraic varieties with application to Abelian varieties, Trans Amer. Math. Soc. 22: 327-482. Awarded the Bôcher Memorial Prize by the American Mathematical Society in 1924. A translation, with minor modifications, of the memoir awarded the Prix Bordin by the Académie des Sciences, Paris, for the year 1919; for announcement, see C. R. Acad. Sci. Paris 169: 1200-1202; Bull. Sci. Math. 44: 5-7.

1923

[25] Continuous transformations of manifolds, Proc. Nat. Acad. Sci. U.S.A. 9: 90-93.

[26] Progrès récents dans la théorie des fonctions abéliennes, Bull. Sci. Math. 47: 120-128.

[27] Sur les intégrales de seconde espéce des variétés algébriques, C. R. Acad. Sci. Paris 176: $941-943$.

[28] Report on curves traced on algebraic surfaces, Bull. Amer. Math. Soc. 29: 242-258. 1924

*[29] L'Analysis situs et la géométrie algébrique, Gauthier-Villars, Paris. (Collection de Monographies publiée sous la Direction de M. Émile Borel.) Based in part on a series of lectures given in Rome in 1921 under the auspices of the Institute of International Education; also on research conducted under the auspices of the American Association for the Advancement of Science. Nouveau tirage, 1950. MR 11, 456.

[30] Sur les intégrales multiples des variétés algébriques, J. Math. Pures Appl. (9) 3: $319-343$.

1925

[31] Intersections of complexes on manifolds, Proc. Nat. Acad. Sci. U.S.A. 11: 287-289.

[32] Continuous transformations of manifolds, Proc. Nat. Acad. Sci. U.S.A. 11: 290-292. 1926

*[33] Intersections and transformations of complexes and manifolds, Trans. Amer. Math. Soc. 28: $1-49$.

[34] Transformations of manifolds with a boundary, Proc. Nat. Acad. Sci. U.S.A. 12: $737-739$.

1927

[35] Un théorème sur les fonctions abéliennes, In Memoriam N. I. Lobatschevskiì, Kazan', Glavnauka, pp. 186-190.

*[36] Manifolds with a boundary and their transformations, Trans. Amer. Math. Soc. 29: $429-462,848$.

[37] Correspondences between algebraic curves, Ann. of Math. (2) 28: 342-354.

[38] The residual set of a complex on a manifold and related questions, Proc. Nat. Acad.

Sci. U.S.A. 13: 614-622, 805-807.

[39] On the functional independence of ratios of theta functions, Proc. Nat. Acad. Sci. U.S.A. 13: 657-659.

1928

[40] Transcendental theory: Singular correspondences between algebraic curves: Hyperelliptic surfaces and Abelian varieties, Selected Topics in Algebraic Geometry, vol. 1. Chaps. 15-17, pp. 310-395; NRC Bulletin no. 63, Report of the Committee on Rational Transformations of the National Research Council, Washington, D. C.

[41] A theorem on correspondences on algebraic curves, Amer. J. Math. 50: 159-166.

*[42] Closed point sets on a manifold, Ann. of Math. (2) 29: 232-254.

1929

[43] Géométrie sur les surfaces et les variétés algébriques, Mém. Sci. Math., fasc. 40, Gauthier-Villars, Paris.

[44] Duality relations in topology, Proc. Nat. Acad. Sci. U.S.A. 15: 367-369.

1930

[45] Topology, Amer. Math. Soc. Colloq. Publ., vol. 12, Amer. Math. Soc., Providence, R.I. See [100].

[46] Les transformations continues des ensembles fermés et leurs points fixes, C. R. Acad. Sci. Paris 190: 99-100. 
[47] (with W. W. Flexner), On the duality theorems for the Betti numbers of topological manifolds, Proc. Nat. Acad. Sci. U.S.A. 16: 530-533.

*[48] On transformations of closed sets, Ann. of Math. (2) 31: 271-280.

1931

[49] On compact spaces, Ann. of Math. (2) 32: 521-538.

1932

[50] On certain properties of separable spaces, Proc. Nat. Acad. Sci. U.S.A. 18: 202-203.

*[51] On separable spaces, Ann. of Math. (2) 33: 525-537.

*[52] Invariance absolue et invariance relative en géométrie algébrique, Mat. Sb. 39: 97-102.

1933

*[53] On singular chains and cycles, Bull. Amer. Math. Soc. 39: 124-129.

[54] (with J. H. C. Whitehead), On analytical complexes, Trans. Amer. Math. Soc. 35: $510-517$.

*[55] On generalized manifolds, Amer. J. Math. 55: 469-504.

1934

[56] Elementary one- and two-dimensional topology, A course given by Prof. Lefschetz, Spring 1934; notes by H. Wallman, Princeton University, Princeton, N.J. (mimeographed).

*[57] On locally connected and related sets, Ann. of Math. (2) 35: 118-129.

1935

[58] Topology, Lectures 1934/35; notes by N. Steenrod and H. Wallman, Princeton University, Princeton, N.J. (mimeographed).

[59] Algebraicheskaîa geometriia: metody, problemy, tendentsii, Trudy Vtorogo Vsesoiuznogo Matematicheskogo S"ezda, vol. 1, (Leningrad, 24-30 June 1934), Leningrad-Moscow, pp. 337-349. An invited address at the Second All-Union Mathematical Congress.

*[60] Chain-deformations in topology, Duke Math. J. 1: 1-18.

[61] Application of chain-deformations to critical points and extremals, Proc. Nat. Acad.

Sci. U.S.A. 21: 220-222.

[62] A theorem on extremals. I, II, Proc. Nat. Acad. Sci. U.S.A. 21: 272-274, 362-364.

[63] On critical sets, Duke Math. J. 1: 392-412. 1936

[64] On locally-connected and related sets (second paper), Duke Math. J. 2: 435-442.

*[65] Locally connected sets and their applications, Mat. Sb. 1: 715-717; A contribution to the First International Topological Conference, September 1935.

[66] Sur les transformations des complexes en sphères, Fund. Math. 27: 94-115.

[67] Matematicheskaia deiatel'nost' v Prinstone, Uspehi Mat. Nauk 1: 271-273.

[68] Lectures on algebraic geometry 1936/37; notes by M. Richardson and E. D. Tagg, Princeton University, Princeton, N.J., (planographed).

[69] Algebraicheskaîa geometriî, Uspehi Mat. Nauk 3: 63-77.

[70] The role of algebra in topology, Bull. Amer. Math. Soc. 43: 345-359. Address of retiring president of the American Mathematical Society.

*[71] On the fixed point formula, Ann. of Math. (2) 38: 819-822. 1938

[72] Lectures on algebraic grometry. Part II, 1937/38, Princeton Univ. Press, Princeton, N.J. (planographed).

[73] On chains of topological spaces, Ann. of Math. (2) 39: 383-396.

74] On locally connected sets and retracts, Proc. Nat. Acad. Sci. U.S.A. 24: 392-393.

[75] Sur les transformations des complexes en sphères (note complémentaire), Fund. Math. 31: 4-14.

*[76] Singular and continuous complexes, chains and cycles, Mat. Sb. 3: 271-285. 1939

[77] On the mapping of abstract spaces on polytopes, Proc. Nat. Acad. Sci. U.S.A. 25: 49-50.

1941

[78] Abstract complexes, Lectures in Topology (University of Michigan Conference, 1940), Univ. of Michigan Press, Ann Arbor, Mich., Oxford Univ. Press, London, pp. 1-28. MR 3, 132.

[79] Algebraic topology, Amer. Math. Soc. Colloq. Publ., vol. 27, Amer. Math. Soc., Providence, R.I. MR 4, 84.

[80] Topics in topology, Ann. of Math. Studies, no. 10, Princeton Univ. Press, Princeton, N.J.; Oxford Univ. Press, London; Second printing, 1951. MR 4, 86. 
[81] Emile Picard (1856-1941): Obituary, Amer. Philos. Soc. Yearbook 1942, pp. 363-365.

$$
1943
$$

[82] Introduction to non-linear mechanics, by N. M. Krylov and N. N. Bogoljubov; a free translation by'S. Lefschetz of excerpts from two Russian monographs; Ann. of Math. Studies, no. 11, Princeton Univ. Press. Princeton, N.J.; Oxford Univ. Press, London. MR 4, 142.

[83] Existence of periodic solutions for certain differential equations, Proc. Nat. Acad. Sci. U.S.A. 29: 29-32. MR 4, 142.

[84] Lectures on differential equations, Ann. of Math. Studies, no. 14, Princeton Univ. Press, Princeton, N.J.; Oxford Univ. Press, London. MR 8, 68.

$$
1949
$$

[85] Introduction to topology, Princeton Math. Series, no. 11, Princeton Univ. Press, Princeton, N.J.; Oxford Univ. Press, London; A work originating from a short course delivered in 1944 before the Institute of Mathematics of the National University of Mexico. MR 11, 193.

[86] Theory of oscillations, by A. A. Andronov, A. A. Vitt and C. E. Haìkin; English language edition edited under the direction of S. Lefschetz. Princeton Univ. Press, Princeton, N.J. MR 10, 535

[87] Scientific research in the U.S.S.R.: Mathematics, Amer. Acad. Polit. Soc. Sci. Ann. 263: $139-140$.

$$
1950
$$

[88] Contributions to the theory of nonlinear oscillations, edited by S. Lefschetz, Ann. of Math. Studies, no. 20, Princeton Univ. Press, Princeton, N.J.; Oxford Univ. Press, London.

[89] The structure of mathematics, American Scientist 38: 105-111. MR 11, 305.

1951

[90] Numerical calculations in nonlinear mechanics, Problems for the Numerical Analysis of the Future, Nat. Bur. Standards Appl. Math. Ser., no. 15, U.S. Government Printing Office, Washington, D.C., pp. 10-12. MR 13, 162.

1952

[91] Contributions to the theory of nonlinear oscillations. Vol. 2, edited by S. Lefschetz, Ann. of Math. Studies, no. 29, Princeton Univ. Press, Princeton, N.J.; Oxford Univ. Press, London.

[92] Notes on differential equations, Contributions to the Theory of Nonlinear Oscillations, vol. 2, Princeton Univ. Press, Princeton, N.J.; Oxford Univ. Press, London, pp. 61-73. MR 14, 557.

$$
1953
$$

[93] Algebraic geometry, Princeton Math. Series, no. 18, Princeton Univ. Press, Princeton, N.J. MR 15, 150.

[94] Algunos trabajos recientes sobre ecuaciones diferenciales, Memoria de Congreso Cientifico Mexicano, vol. 1, U.N.A.M., Mexico, pp. 122-123.

[95] Las grades corrientes en las matemáticas del siglo XX, Memoria de Congreso Cientifico Mexicano, vol. 1, U.N.A.M., Mexico, pp. 206-211.

$$
1954
$$

[96] Russian contributions to differential equations, Proc. Sympos. Nonlinear Circuit Analysis (New York, 1953), Polytechnic Institute of Brooklyn, New York, pp. 68-74. MR 16, 433.

*[97] Complete families of periodic solutions of differential equations, Comment. Math. Helv. 28: 341-345. MR 16, 822 .

[98] On Liénard's differential equation, Wave Motion and Vibration Theory, Proc. Sympos. Appl. Math., vol. 5, McGraw-Hill, New York, pp. 149-153. MR 15, 958.

$$
1956
$$

[99] On a theorem of Bendixson, Bol. Soc. Mat. Mexicana (2) 1: 13-27. MR 18, 481.

[100] Topology, 2nd ed., Chelsea, New York. (Cf. [45]).

$$
1957
$$

[101] On coincidences of transformations, Bol. Soc. Mat. Mexicana (2) 2: 16-25. MR 20 $\# 1320$.

[102] The ambiguous case in planar differential systems, Bol. Soc. Mat. Mexicana (2)

2: 63-74 MR 21 \# 2793.

[103] Witold Hurewicz: in memoriam, Bull. Amer. Math. Soc. 63: 77-82. MR 19, 108.

[104] Sobre la modernizacion de la geometria, Rev. Mat. 1: 1-11. 
[105] Differential equations: Geometric theory, Pure and Appl. Math., vol. 6, Interscience, New York. (Cf. [116]). MR 20 \# 1005.

[106] On the critical points of a class of differential equations, Contributions to the Theory of Non-Linear Oscillations, vol. 4, Ann. of Math. Studies, no. 41, Princeton Univ. Press, Princeton, N.J., pp. 19-28. MR 21 \#2794.

[107] Liapunov and stability in dynamical systems, Bol. Soc. Mat. Mexicana (2) 3: 25-39. MR 20 \# 4685.

[108] The stability theory of Liapunov, Lecture Series, no. 37, University Institute for Fluid Dynamics and Applied Mathematics, College Park, Md.

1960

[109] Controls: an application of the direct method of Liapunov, Bol. Soc. Mat. Mexicana (2) 5: 139-143. MR 24 \# A2730.

[109a] On automatic controls, IRE Trans. CT-7: 474-475. MR 22 \# 10866.

[110] Algunas consideraciones sobre las matemáticas modernaś, Rev. Un. Mat. Argentina 20: $7-16$.

[111] Resultados nuevos sobre casos criticos en ecuaciones diferenciales, Rev. Un. Mat. Argentina 20: 122-124. MR 26 \# 6516.

1961

[112] The critical case in differential equations, Bol. Soc. Mat. Mexicana (2) 6: 5-18. MR 24 \# A3354.

[113] Geometricheskaia teoriia differentsial'nykh Uravnenii, IL, Moscow. (Transl. of [105]). MR 22 \# 12257.

[114] (with J. P. LaSalle), Stability by Liapunov's direct method, Math. in Sci. and Engineering, vol. 4, Academic Press, New York. MR 24 \# A2712.

[115] (with J. P. LaSalle), Recent Soviet contributions to ordinary differential equations and non-linear mechanics, J. Math. Anal. Appl. 2: 467-499. MR 23 \# A2300.

1962

[116] Differential equations: Geometric theory, 2nd rev. ed., Pure and Appl. Math., vol. 6, Interscience, New York. MR 27 \# 3864.

[117] (edited with J. P. LaSalle), Recent Soviet contributions to mathematics, Macmillan, New York. MR 29 \# 4656.

1963

[118] On indirect automatic controls, Analytic Methods in the Theory of Non-Linear Vibrations (Proc. Internat. Sympos. Non-Linear Vibrations, vol. 1, 1961), Izdat. Akad. Nauk Ukrain. SSR, Kiev, pp. 23-24. MR 28 \# 2013.

[119] Some mathematical considerations on nonlinear automatic controls, Contributions to Differential Equations, vol. 1, Interscience, New York, pp. 1-28. MR 27 \# 5010.

[120] Elementos de topologia, Universidad Nacional Autónoma de México.

[121] (edited with J. P. LaSalle), Proceedings of International Symposium on Nonlinear Differential Equations and Nonlinear Mechanics (Colorado Springs, 1961), Academic Press, New York. MR 26 \# 3952.

[122] Stability of nonlinear automatic control systems, Math. in Sci. and Engineering, vol. 13, Academic Press. MR 30 \# 5860.

1965

[123] Liapunov stability and controls, J. Soc. Indust. Appl. Math. Ser. A. 3: 1-6. MR 33 \# 538?

[124] Planar graphs and related topics, Proc. Nat. Acad. Sci. U.S.A. 54: 1763-1765. MR 32 \# 6438.

[125] Recent advances in the stability of nonlinear controls, SIAM Rev. 7: 1-12. MR 31 \# 3686.

[126] Some applications of topology to networks, Proc. Third Annual Allerton Conference on Circuit and System Theory, University of Illinois, Urbana, Ill., pp. 1-6. MR 40 \# 1217. 1966

[127] Stability in dynamics, William Pierson Field Engineering Lectures, March 3, 4, 10, 11, 1966, Princeton University School of Engineering and Applied Science.

[127a] (with J. P. LaSalle), Zarys teorii stabilności Lapunowa i jego metody bezpośredniej (Polish) (Transl. of [114]). MR 33 \# 6051.

[128] Stability of nonlinear automatic control systems (Russian), "Mir", Moscow. (Transl. of [122]). MR 37 \#1723.

[128a] Geometric differential equations: Recent past and proximate future, Proc. Internat. 
Sympos. Differential Equations and Dynamical Systems (Mayaguez, P.R., 1965), Academic Press, New York, pp. 1-14. MR 36 \# 5437.

1968

[129] On a theorem of Bendixson, J. Differential Equations 4: 66-101. MR 36 \# 2879.

[130] A page of mathematical autobiography, Bull. Amer. Math. Soc. 74: 854-879. MR 39 \# 2148.

1969

[131] The Lurie problem on nonlinear controls, Lectures in Differential Equations, vol. 1, Van Nostrand Reinhold, New York, pp. 9-19. MR 39 \# 3857.

[132] The early development of algebraic geometry, Amer. Math. Monthly 76: 451-460. MR 39 \# 4155.

[133] Luther Pfahler Eisenhart, 1876-1965: A biographical memoir, Nat. Acad. Sci. U.S.A., Biographical Memoirs 40:69-90.

[134] Severi's theory of the base for an algebraic surface, J. Analyse Math. 23: 215-225. MR 43 \# 1981.

[135] Selected topics in algebraic geometry, Chelsea, New York.

[136] Selected papers, Chelsea, New York (Contains articles identified by *).

\section{Partial list of volumes Dedicated to SOLOMON LeFSChETZ}

[1A] Algebraic geometry and topology, Princeton Univ. Press, Princeton, N.J., 1957.

[2A] Differential equations and dynamical systems, Proc. Internat. Sympos. (University of Puerto Rico), Academic Press, New York, 1967.

[3A] Journal of Mathematical Analysis and Applications. Volume 10, 1965.

Current address (Lawrence Markus): Department of Mathematics, University of Minnesota, Minneapolis, Minnesota 55455 